\section{Neues Onlineportal Krebs-Zweitmeinung}

- Ab sofort können Patienten fast aller Krebsarten den Onlinedienst www.krebszweitmeinung.de nutzen. Betreiber des neuen Zweitmeinungsportals ist die Health Management Online AG (HMO AG), die diesen Service in einer knapp dreijährigen Pilotphase zusammen mit der Felix Burda Stiftung bei Darmkrebspatienten entwickelt hat.

Das Verfahren ist einfach: Alles, was der Patient tun muss, ist sich online für den Service anzumelden, ein Case Manager nimmt dann persönlich Kontakt mit ihm auf. „In einem ersten Schritt wird geprüft und bewertet, ob eine Zweitmeinung hinsichtlich Fragestellung und Vollständigkeit der Unterlagen für den jeweiligen Patienten überhaupt infrage kommt. Dieser Vorabservice ist kostenfrei", erklärt Ekkehard Wolf, Case Manager der HMO AG. Wird eine Zweitmeinung als nützlich angesehen, trägt der Case Manager gemeinsam mit dem Patienten die Unterlagen zusammen, überträgt diese in eine OnlineAkte und gibt den Fall an ein anerkanntes und hochspezialisiertes Expertengremium weiter.
Im Fokus des Zweitmeinungsservice steht die Verbesserung der Heilungschancen. Besonders sinnvoll ist die Zweitmeinung für Krebspatienten, bei denen die Situation nicht eindeutig zu beurteilen ist und mehrere Therapiewege infrage kommen. Auch Patienten, die sich unsicher sind, welche Therapieoption die richtige für sie ist, sollten den Zweitmeinungsservice nutzen.

Die Erstellung der Zweitmeinung durch ein führendes Tumorboard einer deutschen Universitätsklinik oder eines Krebszentrums sowie der Vermittlungsservice und die sechsmonatige persönliche Nachbetreuung durch die HMO AG kostet für Patienten aus Deutschland einmalig 379 EUR. Von jedem Darmkrebs-Fall geht eine Spende an den Hilfsfonds Darmkrebs der Felix Burda Stiftung. Zudem wird ein Härtefall-Fonds installiert. Die HMO $A G$ verhandelt derzeit mit mehreren Kassen, um eine Kostenübernahme für möglichst alle Patienten zu erreichen.

Nach Informationen der Health Management Online AG, Oberhaching
Silodosin billiger

Der Preis für Urorec (Silodosin) zur Therapie der Benignen Prostatahyperplasie wurde gesenkt. Diese Maßnahme ist die Reaktion auf die zum 1.12.2011 durch den GKV-Spitzenverband erfolgte Festlegung der Festbeträge für Silodosin, nachdem der Gemeinsame Bundesausschuss die Substanz bei den Alpharezeptorenblocker, Festbetragsgruppe 2, eingruppiert hatte. Silodosin ist bis 2020 patentgeschützt. Der nun erteilte Festbetrag orientiert sich aber am hochgenerischen Tamsulosin und liegt so sehr niedrig. Merckle Recordati hat daher beschlossen, die Herstellerabgabepreise für Urorec deutlich um durchschnittlich über $50 \%$ zu senken.

Bei Verordnung von 100 Tabletten Urorec ${ }^{\circ}$ $8 \mathrm{mg}$ oder $4 \mathrm{mg}$ (d.h. der Therapie für ein Quartal) ist jetzt eine Zuzahlung von 14,49 EUR (statt bisher max. 8,97 EUR) zusätzlich zur gesetzlichen Rezeptgebühr von 5 EUR notwendig. Das Verordnungsbudget des Arztes und damit die Ausgaben der GKV werden nur mit dem Festbetrag, also Tagestherapiekosten von 36 Cent bei der 8-mg-Dosierung oder 33 Cent bei der 4-mg-Dosierung, belastet. Bezogen auf eine N-3-Packung Urorec $8 \mathrm{mg}$ heißt dies 36,38 EUR statt bislang 89,68 EUR.

Nach Informationen von

Merckle-Recordati, Ulm

\section{Nr. 1 bei Blasenentzündung}

Bei Harnwegsinfektionen vertrauen Apotheker auf die Heilkraft der Bärentraube wie eine aktuelle OTC-Studie der PharmaRundschau zu rezeptfreien Präparaten aus der Apotheke zeigt. Insgesamt wurden 19 Mittel bewertet. In der Kategorie "Urologika" erhielt das Bärentraubenblätter-Produkt Cystinol akut ${ }^{\oplus}$ Dragees die meisten Empfehlungen der befragten Apothekenmitarbeiter.

Um die Beschwerden einer Blasenentzündung schnell zu beseitigen und Komplikationen zu vermeiden, hat die Bekämpfung der eingedrungenen Errger oberste Priorität. Der im Präparat enthaltene Wirkstoff Arbutin tötet die Bakterien schnell und zuverlässig ab und die gereizte Blasenschleimhaut kann sich erholen. Zusätzlich verhindern Tannine das Anheften der Bakterien an der Blasenwand und damit auch deren Vermehrung. Die Behandlung einer unkomplizierten Blasenentzündung mit Cystinol akut ${ }^{\oplus}$ macht eine Antibiotikatherapie in den meisten Fällen überflüssig.

Nach Informationen von

Rottapharm|Madaus, Köln
Schaper \& Brümmer, Salzgitter
Nach Informationen von 